\title{
Summary of the RHIC Retreat 2007
}

\author{
F. Pilat, C. Gardner, C. Montag, T. Roser
}

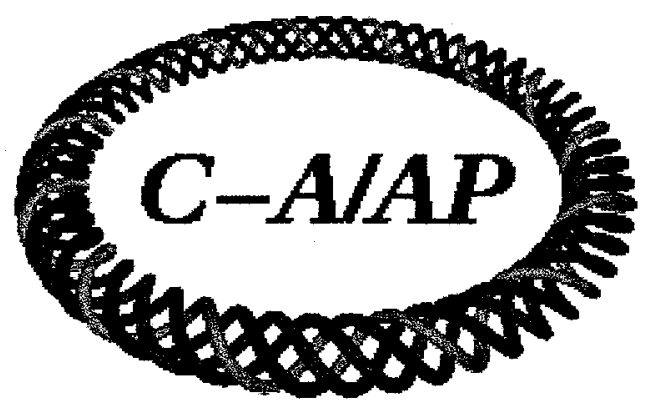

\section{Collider-Accelerator Department Brookhaven National Laboratory Upton, NY 11973}

Notice: This document has been authorized by employees of Brookhaven Science Associates, LLC under Contract No. DE-AC02-98CH10886 with the U.S. Department of Energy. The United States Government retains a nonexclusive, paid-up, irrevocable, world-wide license to publish or reproduce the published form of this document, or allow others to do so, for United States Government purposes. 


\title{
C-AD Note 285
}

\section{Summary of the RHIC Retreat 2007}

\author{
F.Pilat, C. Gardner, C. Montag, T. Roser
}

September 18, 2007
1. Introduction
2. Overview of Run-7
3. Preparation for deuteron-gold operations in Run-8
4. Preparation for polarized proton operations in Run- 8
5. Machine availability and system reliability
6. New developments and future plans

\section{Introduction}

The RHIC Retreat 2007 took place on July 16-17 2007 at the Foxwoods Resort in CT, about 3 weeks after the end of the RHIC Run-7. The goal of the Retreat is traditionally to plan the upcoming run in the light of the results from the previous one, by providing a snapshot of the present understanding of the machine and a forum for free and frank discussion. A particular attention was paid to the challenge of increasing the time at store, and the related issue of system reliability. An interesting Session covered all new developments aimed to improve the machine performance and luminosity.

In Section 2 we summarize the results from Run-7 for RHIC and the injectors and discuss the present objectives of the RHIC program and performance. Sections 3-6 are summaries of the Retreat sessions focused on preparation for deuteron-gold and polarized protons, respectively, machine availability and new developments.

The overall agenda, list of speakers and copy of all Retreat presentations can be found at: http://www.c-ad.bnl.gov/RHIC/retreat2007

\section{Program goals and overview of Run-7}

Run-7 consisted in 13 weeks of $\mathrm{Au}-\mathrm{Au}$ operations at $100 \mathrm{GeV} / \mathrm{u}$ produced twice the total delivered luminosity in Run-4 Au-Au operations. The achieved average store luminosity of $12 \times 10^{26} \mathrm{~cm}^{-2} \mathrm{sec}^{-1}$ exceeded the RHIC enhanced parameter goal of $8 \times 10^{26} \mathrm{~cm}^{-2} \mathrm{sec}^{-1}$. There was no progress towards achieving the goal of $60 \%$ (or $100 \mathrm{~h} /$ week) of time at store. $48 \%$ time at store in Run-7 was only slightly higher than Run- 6 and lower than the mid 50\% achieved in Run-4 and Run-5. 
A comprehensive analysis of RHIC performance goals for Run- 8 and beyond can be found in the latest version of the performance document.

http://www.rhichome.bnl.gov/RHIC/Runs/RhicProjections.pdf

On the basis of what was achieved in the past and improvement plans discussed at the Retreat the immediate goals for Run- 8 are an average store delivered luminosity of $14 \times 10^{28} \mathrm{~cm}^{-2} \mathrm{sec}^{-1}$ for $\mathrm{d}-\mathrm{Au}$ operations (increase of about a factor $4 \mathrm{w}$. r. to Run-3) and of $40 \times 10^{30} \mathrm{~cm}^{-2} \mathrm{sec}^{-1}$ for polarized proton operations. (increase of a factor $2 \mathrm{w}$. r. to Run-6).

The goal for Run-7 was to increase the number of bunches to 111 and the bunch intensity if feasible, in order to reach an average and peak store luminosity of $8 \times 10^{26} \mathrm{~cm}^{-2} \mathrm{sec}^{-1}$ and a $30 \times 10^{26} \mathrm{~cm}^{-2} \mathrm{sec}^{-1}$, respectively. Of the 5 weeks of set-up, 2 were lost on the HX20 heat exchanger problem, so effectively only 2 weeks of set-up were needed from when both rings were at operating temperature. Physics was declared on March 26, with 51 bunches and a peak luminosity of $14 \times 10^{26} \mathrm{~cm}^{-2} \mathrm{sec}^{-1}$. The number of bunches was progressively raised to the target of 111 but eventually reduced to 103 because of beam blow-up and associated losses along the bunch train at transition. The configuration eventually used in operation was 103 bunches with 2 gaps to alleviate instabilities at transition. Approximately midway through the run we reversed the orientation of both experimental magnetic fields in the detectors. Pressure recovery was fast in IR8 and slower in IR6, but the average luminosity never reached the level prior to the magnet change (a fact that still needs explanation).

Longitudinal stochastic cooling was made operational in the yellow ring for the second part of the run with very good results: a 3-fold increase in the beam lifetime, bringing the beam decay in yellow to the burn off rate, and more beam confined in the center bucket. The net effect on integrated luminosity has been estimated to be $\sim 10-20 \%$.

Throughout the run, the average bunch intensity has been $1.12 \times 10^{9}$ in blue and $1.09 \times$ $10^{9}$ in yellow and the average ramp efficiency $93.7 \%$ in blue and $90.6 \%$ in yellow.

In Run-7 the injectors overall exceeded the requirements for gold beam intensity without compromising longitudinal and transverse emittance. The measurements confirmed the average correlation of intensities of AGS and Booster late, with $\sim 50 \%$ transfer efficiency. The main ingredients for improved performance of the injectors were:

- $\mathrm{Au} 31+$ beam delivered from Tandem (instead of 32+)

- Better BtA stripping foil $(31+$ to $77+)$ that provided lower energy spread and loss with higher intensity

- AGS RF merge instead of the debunch-rebunch 'gymnastics' to go from 24 to 4 bunches

- Better and intensity driven tuning strategies

The goal of the polarized proton development (run parasitically to the RHIC and NSRL programs) was primarily to explore the setup with both AGS tunes close to the 9 integer with a $14 \%$ cold snake and the warm snake at its nominal setting. Additional goals were the understanding of polarization losses in the early and slow part of the ramp, and the acquisition of ORM data in AGS. The results were encouraging in that polarization level similar to Run-7 were demonstrated but more development work is necessary to increase AGS polarization to $70 \%$. 
It was stressed that resources are needed for the injector systems upkeep, particularly in the following areas:

- Magnet cooling

- BtA trajectory stability

- Booster orbit correctors

- BtA multiwires

- Signals from Booster instrumentation

Progress was made on modeling, instrumentation and logging of injectors' data. Logging of AtR flags, trajectories, IPM data, tune meter, AGS orbits and WCM were operational by the end of the run and more is planned for the next run.

STAR fully achieved its goals in data collecting for Run-7, both for minimum bias and rare triggers events. Other accomplishments in the run were:

- DAQ1000 (TPX is now on the DAQ monitor page)

- Commissioning and debugging of the Forward Meson Spectrometer (FMS)

- Test of the Muon Test Detector (MTD)

- Preparation for installation in Run-8 of the Time Of Flight system (TOF)

- Installation and some data taking with the Heavy Flavor Tracker (HFT)

The average production DAQ running/day during Run- 7 was $7 \mathrm{~h}$ (minimum of $0 \mathrm{~h}$, maximum of $16 \mathrm{~h}$ ).

- The STAR goals for Run- 8 are d-Au operations, PP at $100 \mathrm{GeV}, 3$ days of pp2pp running, and commissioning of $250 \mathrm{GeV}$, the latter contingent to achieving the physics goals of the previous operation modes. The requirement for $\mathrm{d}-\mathrm{Au}$ is $120 \mathrm{nb}-1$ of delivered luminosity. For the PP run the requirement is for both longitudinal and transverse polarization.

STAR has been rolled out during the summer shutdown and rolled back at the end of August. The schedule for STAR maintenance and upgrade work, including the bake out of the pipe, is consistent with starting the cooldown on Nove mber $1^{\text {st }}$.

Additional feedback and comments from STAR included:

- The interaction between Scheduling Physicist (SP) and Run Coordinator (RC) are not always optimal, although most of the times it works out. (The CAD operations group is following up on this issue by clarifying in writing role and responsibility of SP and RC, and line of authorities to the operations staff).

- STAR stresses once again that the organization of the experiment - agreed upon by DoE - requires scheduled maintenance days planned well in advance. STAR commended the improved organization of $\mathrm{CAD}$ maintenance and encourages continuing the planning effort.

- STAR reminds that to change policies that are agreed for the run needs discussion and collective approval. Also, scheduling configuration changes before long weekends should be carefully avoided in the future (example: the change of magnet polarity before Memorial Day weekend).

Phenix achieved a substantial fraction of its physics goals during Run-7. 0.2 billion minimum bias events were recorded. The $813 \mu \mathrm{b}^{-1}$ recorded luminosity was 3.4 times the one recorded in Run-4. Four new systems were tested:

- RXNP (Reaction Plane Detector) 
- MPC-N (The Muon Piston Calorimeter- North)

- TOF-W (Time of Flight - West)

- HBD (Hadron Blind Detector). The west side of the HBD had in fact to be taken out on April $25^{\text {th }}$ but the diagnostics done was essential to fix and prepare the detector for Run-8.

Phenix estimated that the improvement to the vertex due from stochastic cooling was of the order of $16 \%$. Average time in collision, defined as time in which the CDC counted more than $2 \mathrm{KHz}$ ) was $47.5 \%$. The Run-7 performance of the DAQ was up to $5 \mathrm{KHz}$ event rate and $700 \mathrm{Mb} / \mathrm{sec}$ data throughput. With the luminosity delivered initially in the stores, Phenix still had DAQ saturation at the beginning, typically ranging between 6 and 30 minutes).

Other running related issues that need improvement:

- Water cooling

- Air conditioning

- Flammable gas detectors

- Fire alarm at power shutdowns

- Magnet trips

Phenix control room would like access to the machine e-logs for the Phenix shift crews.

\section{Preparation for deuteron-gold operations in Run-8}

The present setup and performance of the Tandem, Booster, and AGS for the delivery of gold ions to RHIC is summarized in [1]. This year we used Au31+ in Booster that gave us $20 \%$ more beam at Booster injection. A new Aluminum-Carbon stripper in the BTA dramatically reduced the longitudinal emittance of the gold beam in the AGS. A new bunch merging on the AGS injection porch eliminated the need to de-bunch and re-bunch the beam there. The present setup gives 1.5 to $1.8 \times 10^{9}$ gold ions per bunch at AGS extraction. The longitudinal emittance is $0.23 \mathrm{eVs}$ per nucleon (per bunch) and the transverse emittance is $10 \pi \mathrm{mm}$ mrad (95\% normalized) in the ATR line.

The setup and performance of the Tandem, Booster, and AGS for Run 3 is summarized in [2]. For the deuteron setup we need a two-to-one bunch merge in Booster with eight transfers to AGS per AGS cycle. The deuteron beam intensity at the end of the TTB line was typically $1.5 \times 10^{11}$ deuterons per pulse with a maximum observed intensity of $2.0 \mathrm{x}$ $10^{11}$. The typical intensity at AGS extraction was $1.3 \times 10^{11}$ deuterons per bunch. The longitudinal emittance of the deuteron beam is estimated to be $0.7 \mathrm{eVs}$ per nucleon at AGS extraction. The maximum transverse emittance at Booster extraction is estimated to be $25 \pi \mathrm{mm} \operatorname{mrad}$ ( $95 \%$ normalized).

The setup and performance of the collider during Runs 7 and 3 are summarized in [3] and [4] respectively. The deuteron beam intensity in RHIC was $1.2 \times 10^{11}$ per bunch. The normalized transverse emittance was $12 \pi \mathrm{mm}$ mrad (95\%) and the bunch length at store was $5 \mathrm{~ns}$.

For Runs 3 and 7 we ran with beta* of 2 and $0.8 \mathrm{~m}$ respectively. The plan is run with $1 \mathrm{~m}$ beta* for Run-8. In order to reduce the stress on the RHIC injection kickers, the nominal 
magnetic rigidity of $\mathrm{Au} 79+$ ions at $\mathrm{RHIC}$ injection has been reduced from $90 \mathrm{Tm}$ (the value used in Run-3) to $86 \mathrm{Tm}$. We also would like to have remote control of the Lambertson magnet position for Run-8.

There is also a plan to implement a scheme to adjust the yellow main dipole to maintain the desired yellow radius around transition.

Negative ions (Au-1) from a pulsed sputter source [1] are accelerated from ground potential to $+14 \mathrm{MV}$ at the center terminal of the Tandem where they pass through a thin ( $2 \mu$-gram $/ \mathrm{cm}^{2}$ graphite) stripping foil. The ions emerge predominately in charge state +12 and are accelerated back to ground potential. A second stripping to charge state +31 occurs in a $15 \mu$-gram $/ \mathrm{cm}^{2}$ graphite foil downstream of Tandem. This charge state survives well in the Booster vacuum and allows sufficient energy gain in Booster to efficiently remove all but two electrons before injection into AGS. In the past we have used charge state +32 in order to stay easily within voltage and current constraints on the Booster main magnet power supply. This year we were able to operate within these constraints with charge state +31 . The result has been a $20 \%$ increase in the number of ions available for Booster injection.

This year we also acquired and tested several $2 \mu$-gram $/ \mathrm{cm}^{2}$ graphite foils formed by laser plasma ablation. These were found to last some three times longer than our standard terminal foils. The plan is to load both Tandems with these new foils. This amounts to 600 foils for the MP7 Tandem and 500 for MP6. The pulse width from the source ranges from 800 to $900 \mu$-seconds. Intensities of $5 \times 10^{9} \mathrm{Au} 31+$ ions per pulse at the end of the TTB line are typical, although intensities twice as high have been achieved. Transport efficiency of the entire line ranges from 80 to $90 \%$.

During Run- 7 the total Tandem downtime was 8.75 hours and for the TTB line was 8.15 hours. Ten foil changes required more than 6 minutes was 10 for a total of 2.86 hours. The MP7 Tandem had to be opened three times during Run 7; MP6 provided Au beam during these times.

The MP6 Tandem is used for the delivery of deuterons. A solid titanium deuteride cathode is used in the source. For Run 8 a higher voltage $(20 \mathrm{kV})$ pulser will be used.

The typical deuteron beam intensity at the end of the TTB line in Run- 3 was $1.5 \times 10^{11}$ deuterons per Tandem pulse with a maximum of $2 \times 10^{11}$. Eight Tandem pulses are required for each AGS cycle to achieve the deuteron bunch intensity desired for RHIC

A horizontal and vertical beam profile monitor must remain inserted in the TTB line to monitor the intensity of the deuteron beam for safety reasons. This monitor intercepts about $15 \%$ of the beam. It has been proposed to reduce this to $7 \%$ and this requires approval by the Radiation Safety Committee.

Any failure requiring that MP6 or MP7 be opened during Run 8 could result in up to five days loss of beam operations. A multi-cathode sputter source is being developed and tested that would allow deuterons and gold ions to be produced from the same Tandem with a switching time of less than 30 minutes.

A new bunch-merging scheme was developed this run and used on the AGS injection porch [1]. The scheme works well and will be used for Run 8. For gold ions, 24 bunches 
are merged into 12 and then into 4 . For deuterons, only a merge from 8 to 4 bunches is required. Switching between the gold and deuteron merges will need to be PPM.

In Booster we need to resurrect the deuteron merge scheme used during Run 3 [2]. This is a merge from two to one bunch done on a short porch during the Booster magnetic cycle. This setup needs some development time as new low-level RF components have been installed since it was last used. The setup also needs to be PPM with the gold setup and with any NSRL setups.

As in Run-3, deuterons in Run 8 will be injected into the blue ring and gold ions into yellow. The main issue here is whether or not to run with the IBS suppression lattice (FODO cells with 92 degrees phase advance) in the yellow ring. The beam experiment performed in Run 7 and predictions indicate a $10-20 \%$ potential benefit for $\mathrm{Au}-\mathrm{Au}$. The benefit for $\mathrm{d}$-Au would be roughly half this.

There are a number of question marks associated with the IBS lattice:

- With 1 meter beta*, is there enough aperture available in the DX magnets

- Matching the lattice arcs and straights results in some beta function distortion.

Can this be reduced to a tolerable level?

- The model of the lattice shows a negative chromaticity jump at transition crossing. This needs to be understood and fixed.

At the time of the retreat it appeared that for the IBS lattice beta* at injection could not be relaxed more than 4 meters with associated possible aperture problems in the DX magnets at injection. A solution with $10 \mathrm{~m}$ beta* at injection is now available.

For deuteron in the blue ring we will use a $1 \mathrm{~m}$ beta* lattice with regular phase advance in the arcs.

Chromaticity needs to be negative below and positive above transition. This implies zero chromaticity near transition; a possible cause of fast transverse instability for short bunches. This is still a performance-limiting factor in RHIC. Jumping the chromaticity set points by 8 instead of 4 units across transition helps a lot. The gamma transition jump can give a fast and unwanted negative chromaticity jump. The Au4 ramp is known to have a positive chromaticity jump according to the model. Electron cloud densities are increased at transition because of the bunch length shortening, and electron clouds have been shown to lower the stability threshold of bunches. 1-2 days of scrubbing at injection may allow accelerating more beam through transition.

The re-bucketing scheme for gold in the yellow ring will be the same as for Run 7 . Rebucketing for deuterons (in blue) depends on the longitudinal emittance. If we need to blow up the emittance as in Run 6 (with protons) then we will not re-bucket. This implies not using the common cavities for gold, and consequently a greater reliability demand on the ring cavities of yellow. Without re-bucketing the bunch length for $1 \mathrm{eVs} / \mathrm{n}$ emittance would be 9 ns.

Longitudinal stochastic cooling has been commissioned and made operational in Run-7 in yellow with the result of effectively eliminating debunching and reducing losses. Stochastic cooling will be implemented in blue, and tested with low intensity deuteron, 
while the plan for yellow is to fix the mechanical failures in situ and improve the operations interface. The microwave link will be used in the Blue ring.

We expect the main intensity limitation for $\mathrm{d}$-Au to be instabilities at transition crossing. The gold bunches with smaller longitudinal emittance have higher peak current than in Run-3 both at injection and transition. In Run-8, the large longitudinal emittance of the deuteron beam and the high gold peak current might create problem at IRs, and/or for transition crossing. If necessary, high intensity $\mathrm{d} / \mathrm{Au}$ beam can be injected for beam scrubbing. The pro is that the most troubled sections will be scrubbed more. The con is that we are not sure that the relevant area of the pipes will get scrubbed to alleviate transition and re-bucketing pressure rise.

Mode switching is an important aspect of deuteron-gold operations. During Run-3 the mode switching time was reduced from 9 to 4 minutes. Some additional streamlining of the Tape sequences is possible, but any further reduction is expected to be modest. Although we have not done any $\mathrm{d}-\mathrm{Au}$ mode switching since Run-3, NSRL operations added a lot of experience in mode switching. In addition, the sequencer much improved from Run-3.

Further action items for Run-8:

- Compartmentalize mode switch tasks. Store Tandem, Booster, and AGS setups in separate areas.

- Modify tape sequences to allow for more round-robin type mode switches in order to accommodate pp setup or NSRL running

- Create a BTA magnet manage

- Identify other actions that can be made PPM

- Establish a list of deuteron and gold setup items that must be checked before terminating a store

- Implement injection replay corrections.

\section{Preparation for polarized proton operations in Run-8}

The goal for the polarized proton run in FY08 is to double the average store luminosity from 20 to $40^{*} 10^{30} \mathrm{~cm}^{-2} \mathrm{sec}^{-1}$, while maintaining the polarization transmission on the RHIC ramp at the near $100 \%$ level. A $10 \%$ improvement can be achieved by reducing the beta function at the interaction points from 1 to $0.9 \mathrm{~m}$. The majority of the luminosity increase, however, has to come from higher bunch intensities.

Several measures are being planned in order to accommodate the larger beam-beam tune spread associated with higher bunch intensities. Correcting the nonlinear chromaticity reduces the tune footprint of the beam; the tune space thus freed can allow for a larger beam-beam tune shift resulting in an increase of the total beam-beam induced tune spread from 0.012 to 0.015 . The nonlinear chromaticity was successfully corrected during Run7; however, the small beam-beam tune shift parameter associated with Au-Au operations did not allow observing of a beneficial effect on luminosity performance.

A large improvement is expected from changing the working point in one ring. Tracking studies performed over the past year predict a dynamic aperture of about 7 
sigmas for tunes below the integer, (27.96/28.95). This is comparable with results for tunes around (28.695/29.685), and considerably better than at the mirror tunes $(28.685 / 29.695)$. We therefore plan to operate the Blue ring at the near-integer working point (27.96/28.95), while the Yellow ring remains at the "good" operational working point $(28.695 / 29.685)$.

Among the operational concerns associated with a working point next to the integer is the increased sensitivity to misalignments and quadrupole errors. The former will result in a two- to three-fold increase in the $10 \mathrm{~Hz}$ orbit jitter amplitude at the IP caused by mechanical triplet vibrations. The now operational IR orbit feedback is currently capable of reducing the jitter amplitude at the IP by a factor three to four, thus resulting in modulated beam-beam offsets of the same magnitude as in previous years without such feedback. Ongoing work on the new Bergoz BPM's may result in a higher feedback gain in the future and therefore a net improvement even at the new working point.

Beam experiments with gold beam at injection have been performed during FY07 to measure the beta-beat at near-integer tunes. The vertical beta-beat measured in the Blue ring turned out to be surprisingly small, around $20 \%$, in comparison to the $40 \%$ in the horizontal plan. This indicates that the horizontal beta-beat is most likely caused by a small number of quadrupoles, and not by random errors of a large number of magnets. Optics measurements and correction algorithms are currently being developed to correct these errors.

Tune feedback will be mandatory to ensure successful operation near the integer, since even small tune changes result in significant orbit and optics distortions due to increased sensitivity at the new working point. While this system was already used during Run-7, some issues remain that need to be resolved before the start of the polarized proton run. These issues have been identified and are currently being worked on. The feedback code is currently being modified to account for the different tune response matrix coefficients at the new working point.

While there was no polarized proton operation in RHIC during FY07, polarized proton setup neverthelesss took place in the AGS. The main goal of these activities was to test a new snake configuration and a higher horizontal tune during the ramp to overcome a weak depolarizing snake resonance. The data analysis is still in progress; the snake setting to be used during the upcoming run will be decided based on these results.

To shorten the bunch length and therefore reduce the hourglass effect, a new $9 \mathrm{MHz}$ cavity will be employed on the ramp. This cavity consists of a shield inside the common cavities to make a $9 \mathrm{MHz}$ resonator. In this configuration, the $9 \mathrm{MHz}$ cavity will be used during acceleration, while the $28 \mathrm{MHz}$ system will take on the role of the storage RF.

The polarimeter will be outfitted with new carbon targets and new detectors for Run-8. A modified target drive with less backlash and hysteresis has been installed in the $\mathrm{pC}$ polarimeter, and new target holders will provide capability to correct for targets that are not straight. The targets themselves are now produced at BNL, at a rate of about one target per day. Besides thin $(25 \mathrm{~nm})$ polarimetry targets, thicker $(50$ and $100 \mathrm{~nm})$ targets are also being produced which are dedicated to beam scanning.

While the $\mathrm{pC}$ polarimeter averages over the axis perpendicular to the scan direction, the jet polarimeter averages over the entire beam in both planes. Normalization of $\mathrm{pC}$ results to jet data therefore requires a correction for this profile dependence. This profile dependence is important since the experiments use the luminosity-averaged polarization, 
while the jet measures the intensity-averaged polarization. Better beam scanning tools should allow for the required measurements during Run-8.

\section{Machine availability and system reliability}

The overall availability of RHIC and injectors - defined as the percent of time at store in collision over calendar time - steadily grew over the early operations years until Run-5, and decreased in Run- 6 and Run- 7.

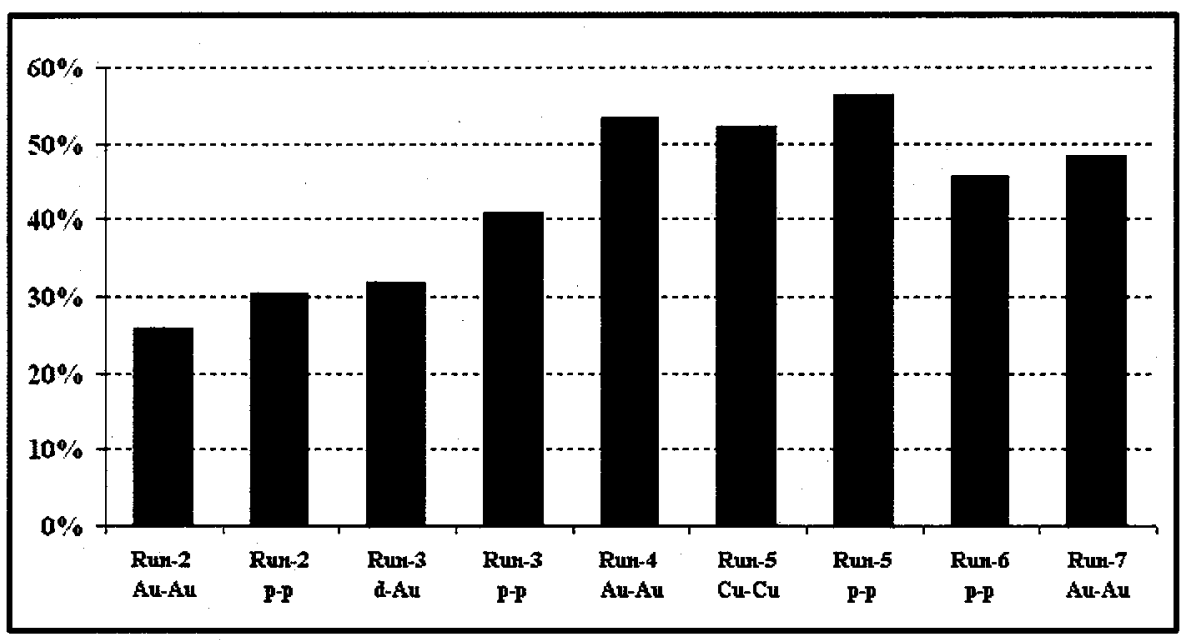

This session was devoted to analyze the causes, and to develop plans towards restoring availability over $50 \%$ in Run- 8 and reaching the $60 \%$ goal, possibly in Run-9. Factors affecting the time at store are:

- Machine set-up time

- Machine development (short term)

- Accelerator Physic s experiments (long term)

- Scheduled maintenance

- System failure

We addressed at the Retreat all these issues and evaluated where improvement is possible. The main contributor to loss of time in collision is system failure: in Run-7, 881 hours out of 3087 refrigerator hours, or $28.5 \%$, were spent in a failed state.

The statistics on failure by systems and by group are summarized here below, respectively: 


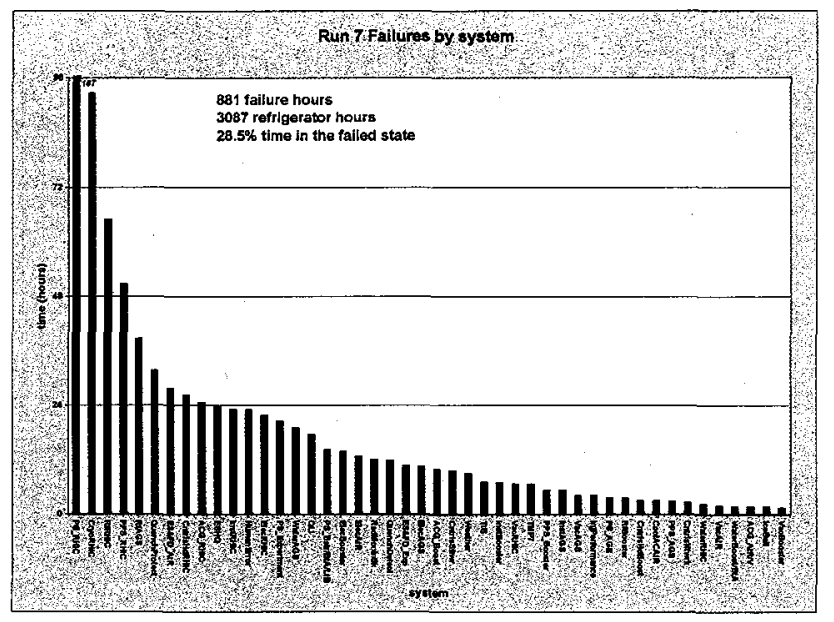

The distribution of charged failure hours per group for the last 4 RHIC Runs is summarized in the table below:

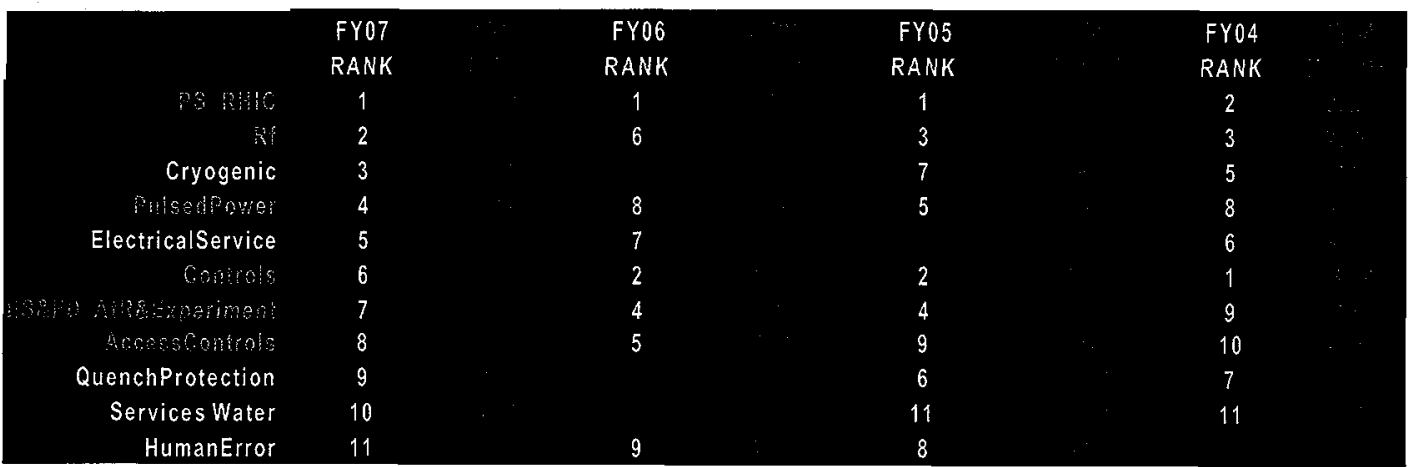

More detailed data on failure of selected systems during Run-7 can be found below. The ration of actual/charged failure hours per system is more than 1 (sometimes significantly) as more than one system can be down at the same time. A severe failure causes interruption of physics program; a mild failure may impact subset of operations; it gets recorded but does not cause physics downtime. The threshold for a recorded instance is 6 minutes. Shorter interruptions are considered resets. It must be noticed that resets down time is not negligible and totaled an integrated $27.8 \mathrm{~h}$ during Run-7, more than a day of running.

\begin{tabular}{|c|c|c|c|c|c|c|c|}
\hline \multirow{2}{*}{\multicolumn{2}{|c|}{ System }} & Charged (h) & $\begin{array}{c}\text { Actual } \\
\text { Severe (h) }\end{array}$ & $\begin{array}{l}\text { Actual } \\
\text { Mild (h) }\end{array}$ & Resets (\#) & $\begin{array}{l}\text { Resets (h) } \\
\text { @ } 3 \text { min per }\end{array}$ & $\begin{array}{c}\text { Ratio } \\
\text { Actual/Charged }\end{array}$ \\
\hline & & 187 & 236 & 5 & 15 & 1 & \\
\hline & m & 107 & 216 & 272 & 44 & 2 & \\
\hline & by & 59 & 80 & 15 & 70 & 4 & \\
\hline & Ontols & 39 & 70 & 39 & 303 & 15 & \\
\hline $88+7$ & An: Expetimon & 38 & 53 & 51 & 33 & 2 & \\
\hline & Accossontrols & 36 & 40 & 25 & $\sim 0$ & 0 & \\
\hline
\end{tabular}

The average failure time in Run-7 has been 6.85 hours/day. No significant correlation has been found with (outside) temperature, and only marginally better correlation with humidity. What the distribution of failure vs. time shows is a quite repeatable pattern of one high failure day followed by another. Possible explanations range from the technical 
(one equipment failure - i.e. a switch - fails and this causes inspections and discovery of other possibly failing switches into the next day) to the sociological (personnel collectively losing confidence that things can run smooth again).

Work to minimize system failure has been analyzed system by system. Operations can minimize the impact and speed the recovery when failure occurs. The following measures can help:

- Reinforcing CAS so that multiple failures can be dealt with in parallel

- Training Siemens Watch for LOTO Together with MCR Operators, so that they can perform LOTO when CAS is busy

- Getting Operators into the field and train them to (only) reset "accelerator" power supplies

- Instructing OC to call in help for CAS when CAS is making a repair and another system goes down

- Instructing $\mathrm{OC}$ to call in help from two groups with knowledge of the e equipment when the cause of a problem is not clear

The factors determining a quick store-to-store turnaround time are a working machine (i.e. no failure), communication among operations staff ( $M C R$, Tandem, experiments), advance preparation (i.e. checklists, operation sequences), optimization and keeping score. During Run-7 an opportunity for speedy $(<1 \mathrm{~h})$ turnarounds arose only in the $27 \%$ of cases. The new factor this year was keeping scores, that is, an unofficial contest among operations crews on who's achieving the fastest turnaround time. Not only this contributed to team work, motivation and faster training of new operators, but it had a significant quantitative effect: the average turnaround time for store-to-store less than $1 \mathrm{~h}$ went down from 35 to 22 after starting keeping score ( 1/4 into the physics run). The conclusion is that the injection set-up for Au-Au appears fully optimized now from the operation perspective, with only 2-3 minutes to be possibly gained on STAR and Phenix optimization at the beginning of the store.

The new methods for optimization of scheduled maintenance discussed at the 2006 RHIC Retreat were implemented during Run-7. The job request system, the more formal approval, better documentation and post-mortem analysis have been successful. The concepts of job ownership and close-out have been more difficult to implement in practice, largely due to a lack of time, or emergent situation, that happen at the end of a maintenance day. The results of better maintenance organization have been very successful for the injectors: accelerators operation on or ahead of schedule, minimum impact of sweeps, more rigorous testing of system. For RHIC, the average time between the start of maintenance and beam in RHIC went down in Run-7 by more than 3 hours in comparison with Run-6. The big issue to be resolved is however the failure ensuing the end of maintenance, so that in some cases the physics program could be started only several hours after the nominal end of a maintenance day. A limited number of these failures can be attributed to QA and other issues related to maintenance but the majority is associated with the general rather high failure level. There are unique issues associated to a maintenance day: expert personnel are overworked and undermanned, the schedule is too busy since maintenance days are often used to continue upgrades and shutdown work not completed in the summer, machine developments in the injectors running at times in 
parallel to maintenance, causing confusion and reduced resources. It is possible to improve by increasing preventive maintenance during the shutdown, by strengthening the quality assurance process, and avoiding set-up and development work during scheduled maintenance days.

The typical maintenance models at large accelerator complexes are schedules, and run the machine till it breaks, or until a critical mass of issues warrants an extended shutdown, with RHIC using the former largely because of the needs from the experiments to have scheduled days for planning of detector upgrades. We discussed a "hybrid" model with a limited number (3-4) of scheduled maintenance/upgrade days interleaved with unscheduled, shorter accesses flexibly scheduled, dedicated to fixing and repair as needed. This model was rejected by the STAR collaboration, for which an advance planning of personnel on site is imperative.

In preparation for the Retreat, all systems, with emphasis on those with higher failure rates, were asked to focus their reports on the plans, manpower and time needed to improve the individual system reliability. The resulting presentations were quite comprehensive and detailed, and only a summary of specific and critical issues will be discussed here. We refer to the Retreat WEB page for the necessary details.

The average of RHIC power supplies (PS) failure hours/week went up from 4 during Run-6 to 8.89 in Run-7. The MTBF of RHIC due to any PS failure and of individual PS's were up accordingly. The leading causes of lost time are the Dynapower IR PS, the main PS, the IR SCE 140A, the 6000A quench switches and the IR SC 300A. The RHIC PS correctors in particular - as a result of a multiyear improvement plan - and the new sextupoles power supplies performed remarkably well.

Performance and problems of every PS have been thoroughly analyzed, including causes of failure and related correcting action for the 2007 shutdown and beyond. I will remind a few high priority items.

A single failure - a problem with the blue main dipole PS voltage loop gain - caused almost 19 hours of downtime: a new voltage signal conditioner board needs to be designed and implemented with high priority. Another important aspect is environmental conditions and the requirement of getting $\mathrm{AC}$ to the RHIC PS service buildings. Much operations time has been lost on PS and QPA faults: with some system modifications these could be turned into warning - to be followed up but not interrupting necessarily operations. This is planned for this shutdown.

The top priorities for PS work are:

- Upgrade of bipolar 150A and 300A, Phase 1

- QPA's

- Main PS

- Yellow quad bus ground fault (spontaneously cleared last run but in need of investigation given the potential risk for downtime)

- Dynapower PS cooling

- Quench detector cleaning and fan replacement

- Air conditioning

Projected manpower and timeline are consistent at the time of the Retreat with cool down on November $1^{\text {st }}$. By removing the major problems the PS system performance should 
improve considerably (If we remove the 3 major problems the MTBF of the overall PS system would increase from 14.75 to $40 \mathrm{~h}$, more that the Run- 5 figure of 30.79 .

The following is a status report on shutdown work:

- The rack-mounted QPA 's will be done only in buildings $2 \mathrm{~B}, 4 \mathrm{~B}, 10 \mathrm{~A}, 6 \mathrm{~B}$ and 12A. The fan fault will become a warning and new fans are getting installed. Lots of other work is being done to the QPA 's. This is roughly half of the service building QPA 's. None of the tunnel QPA 's will get done for this run

- Right now it looks like 11 of the 150A bipolar PS 's and about 6 of the bipolar 300A PS's will be retrofitted by an external company. (It is possible we may get 5 more done by the external company if things go very well) We have identified about 13 bipolar $300 \mathrm{~A}$ 's and 27 bipolar $150 \mathrm{~A}$ 's as problematic. We would like to retrofit all the supplies eventually. As a backup plan we are installing external fans on the front of the bipolar 150A's, which won't get retrofitted. This should help.

- On the mains, the voltage signalconditioning card won't be done for the beginning of the run but will probably be ready sometime during the run. The output circuit compartment will have new hardware installed on the SCR resistors to improve the current sharing. This should be complete in a few days.

- We are hoping to change certain faults into warnings on the IR supplies in the service buildings. The service buildings should get done. This will be close. It is unlikely to have the alcoves done in time.

- We have investigated the ground fault problem we had at $8 \mathrm{~B}$ and it looks like we found a supply that caused the problem

- The cooling is being improved in the rack mount unipolar Dynapower PS 's to help prevent the circuit breaker tripping problem in the future. We are also removing the unipolar Dynapower from the b12-q89-ps slot so we have a bipolar PS there again.

- The spares inventory is being increased.

Failure hours for the electrical systems increased in the last 2runs both in absolute and relative (to total failure hours) terms, if we factor out the time lost of Run- 6 flash event. Over $90 \%$ of failure time can be attributed to 4 problem areas:

- Switch and 208 circuit breaker in 1004-A (2 events)

- Multiple switch and circuit breakers in 1000-P

- Switch in bld. 914

- Cooling tower fan motor in bld. 929

Many corrective actions are being taken:

- 18 Electricians Assigned to C-AD this summer vs. 6 last year

- On going Thermal Inspection of Switches

- Use of torque Wrenches Instituted

- Better understanding of Thermal Effects

- Replace 1000 P 13.8 kV Switches

- Replace Trip units 1000 P Substation

- Replace Switchgear in 914

- Maintenance BMMPS CB's 
- High Resistance Grounding Completed

- Inspection of Stand Alone Motor Starters

- Continuation of Arc Flash Calculations

- Connecting RHIC Bard A/C Units through Isolation Transformers

- 21 New Alcove UPS' s

- 8 year Program to improve Electrical Infrastructure (\$9 million)

- Open Slot for New Power Engineer

The main concerns regarding electrical system in the last Retreat were power dips, the response to the 1006 arc flash, 1004-B CB problem and the AMMPS transformer replacement. Most corrective actions planned there were implemented. 2 new diesel generators for the AGS were not installed for Run-7 but a solution with 2 almost new Diesels will be in place for Run-8.

The planned centralization of spare parts location and increase of the spare inventory will help the recovery from failures.

Failure hours for the RF system went considerably up from the previous years: 111 hours total, 7 for Booster, 29 for AGS and 65 fr RHIC RF systems respectively. One underlying reason (for the RHIC systems) is that the higher beam intensity in RHIC caused problems associated with beam loading in the cavities that took considerable time and effort to understand and deal with. Also note that we did not run the RHIC storage RF system last year. All system failures for the Booster, AGS and RHIC RF have been reviewed and in most cases remedial action has been planned for the shutdown and beyond.

- New tubes for Booster RF are purchased and will be installed this summer

- Efforts are under way to modify Booster driver amplifier to increase FB loop gain at low frequency

- All phase and AGC cables will be replaced this summer for the AGS system

- Analysis of water circuit and cavity parts are in progress for L10, spare parts will be purchased

Most failure modes associated with Booster and AGS are identified and plans are in place to resolve them. An open issue that remains are sporadic synchro problems - responsible for about 39 hours of accumulated failure time - never reproduced by the RF system experts. The planned upgrade of LLRF will likely solve these problems.

Failure modes and associated remedial actions have been identified also for the RHIC HLRF and LLRF. These are the planned system improvements:

- Window comparators to provide fast shutdown for storage systems

- New beam permit chassis to speed up the response

- Low power circulators

- New tubes

- Ongoing work on window for storage system

- Continue development of ferrite tuner for acceleration system

The RF reiterated their wish and need to scheduled maintenance at 2 weeks intervals, not only for repair but also for planned system diagnostics and development. Examples are diagnostics without beam, implementation of needed hardware and software diagnostic 
tools, DSP code changes, requests of special RF configurations for APEX or other developments.

Much work was done on the LLRF both in AGS - that resulted and made possible the very successful bunch merge in AGS in Run-7 - and RHIC in preparation for the future planned LLRF upgrade, a modular and high speed digital system. The staged LLRF upgrade will have the advantages of common hardware across AGS and RHIC, and will improve machine-to-machine synchro.

The following is a status report - at print time - on the improvement implemented in the summer shut down:

- A (fast) permit input will cause the circulating beam to be aborted within microseconds in order to reduce the likelihood of a magnet quench due to debunched beam, should an RF Cavity (PA) trip off.

- Voltage Window comparators are installed on the storage cavities. IF the gap volts fall to less then preset level the PA will be turned off. The action is designed to protect equipment and will be transparent to the beam

- Isolators were installed to protect the 5 watt amplifiers in the Low Level system as many amplifiers were lost during the last run

- Band 2 power tubes were replaced in the Booster

- The RFGL will work out a "procedure" with the help of operations \& maintenance to allow "on demand" access to the Booster or the AGS in order to look for suspected water leaks that have the potential to damage amplifier tubes in a PA.

- Band 2 power tubes were replaced in the Booster

- The RFGL The RFGL will work out a "procedure" with the help of operations \& maintenance to allow "on demand" access to the Booster or the AGS in order to look for suspected water leaks that have the potential to damage amplifier tubes in a PA

The failure modes for the RHIC abort kickers in Run-7 were:

- Pre-fires: one module discharges unilaterally, the other four fire in response ASAP, not synchronized with abort gap

- Unconditioned Triggers: all five modules discharge together, not synchronized with the abort gap

- Spontaneous Capacitor Discharges: as if a "stop charge" occurred with no associated trigger - stop charge turns off the charging mechanism. Damaging if not noticed

12 pre-fires happened in yellow, mostly in the first $1 / 2$ of the run, 18 in blue, mostly in the second $1 / 2$. The distribution among modules is also highly uneven. Y5, B2, and B4 had 7 pre-fires each, contributing to $70 \%$ of total pre-fires. B2 and B4 use thyratron CX1575C, so this will be replaced by $\mathrm{CX} 3575 \mathrm{C}$. Y 5 had 7 pre-fire at beginning, but stayed clean after 4/4, Y1 stayed clean during entire run.

The following planned measures will help:

- Conditioning the high voltage system at higher voltage than operation level

- Keeping modulators on

- Pre-conditioning before beam operation 
- Keeping operating voltage as low as possible

The solution that would likely greatly improve the reliability of the abort systems would be to develop a system that charges up the high voltage modulators on command $4 \mathrm{~ms}$ before the beam abort to avoid pre-fires during long DC hold up. A preliminary feasibility study was performed in 2003 and estimate for the project cost was over 2 MS.

The downtime charged to controls in Run-7 was significantly less than in the previous runs, both absolutely and relatively.

In controls hardware, the distribution of memory errors and WFG resets both peak in the alcoves 7 and 9 consistently with high radiation levels. On the basis of these data the VME chassis in the selected alcoves were replaced with radiation tolerant models: 30 VME chassis have been modified with a radiation tolerant power supply with the result that none of these power supplies have failed to date. Also new radiation resistant memory was tested in 7C for the V115 modules during the latter part of the run in three modules. The unmodified module in same test chassis had 10 errors but no memory upsets detected in modules with the new memory.

At print time the status is the following:

- 100 radiation resistant RAM modules installed in alcove FEC' s.

- $20 \mathrm{VME}$ chassis were upgraded with radiation hardened power supplies

- Error correcting memory installed in all but 10 alcove FEC' $s$.

All failures of controls software were carefully analyzed. Out of a total of 21 events analyzed, about $1 / 2$ have been fully understood and a solution exists. The other are still under investigation. The main activity in controls software that have taken place so far during the shutdown are:

- Booster controls upgrades to Main Magnet and Orbit Correction system

- A private subnet for operational FEC' $s$ is being investigated and tested. Availability increased when a private subnet was implemented for the Quench Detection FEC' $s$.

- A series of disaster recovery drills were conducted. These included a switchover from the primary RAID system to the hot backup RAID system in building 923 . System administrators are evaluating the lessons learned.

- The Operations RAID system was exercised/stressed in order to uncover weaknesses. A faulty hardware component was identified and replaced in the RAID chassis.

The down time due to access controls in Run-7 was lower than in previous runs, and the decreasing number PASS and relay of failure mirror that. The upgrade for gate 4GE2, a solid gate with magnetic switch locks - a prototype for a more general gate upgrade was successful: the new device caused no faults or sweep losses. Responsibility for the maintenance and repair of the chipmunks was handed over to the Instrumentation group. $A$ series of upgrades for the ACS is planned:

- Convert BOOSTER, LINAC, and AGS from relay to PLC based system (timescale 1 year)

- Replace mechanical switches with magnetic type switches (2 years)

- Replace Electric strikes with Electro-Magnetic locks (2 years) 
- RHIC PASS Screen Data update speed improvements (Protocol Elimination) ( 6 months)

- Iris Recognition Controlled Access Upgrade (in progress)

In order to focus on priorities we limited the discussion of instrumentation to BPM's, IPM and $\mathrm{BBQ}$ (tune tracker).

IN 2003-2004 all modules were relocated from the ring to the alcoves and service buildings. 175/750 boards had failed for various reasons. Over 100 digital sections that were damaged due to radiation were replaced. In 2005 a Task force reviewed bottom to top the entire system. Many improvements were implemented including relay removal in the signal path, addition of a two-stage gain circuit, many code/ADO improvements. In 2006 the system underwent a complete recalibration to remove position dependency on time. Only 1 IFE failed. Despite all that, over 100 units failed over the course of last run. The completely random failure distribution was nearly all attributed to a high-speed optocoupler well beyond its MTBF. It may have been weaken by radiation when the units were above the beam pipe. This high-speed opto is expensive, obsolete - and electrically unnecessary. We modified a board and tested it with beam - the HS opto was removed and replaced with a piece of wire and we replaced the other 17 low speed optos. The opto removal does not require any Altera code changes. The BPMs are in a much cooler area than they were initially installed. This modification is much simpler than the relay removal effort. The board modification is done off site - $\$ 11$ per board. More than $90 \%$ returned are working. The goal of the shutdown is to repair all bad units, and to modify all units (750) in the alcoves. Also, we should review the high level code to filter out bad data from the BPMs.

The IPM in blue failed during startup. The failure was attributed to insufficient protection of the high voltage and vacuum. Chassis was added and tied into controls. The microchannel plates are of "reduced performance". Prototyping is in the works for new vacuum and radiation hard design.

RHIC IPM Shutdown work consists of evaluation of what interlocks are present and to add more if needed. The micro-channel plates are on order, not here yet. The vacuum group drives the repair schedule. (Lack of vacuum isolation valves prevents quick turn around and long sections have to be baked if the IPMs are vented.) The most likely plan is to install a prototype as a $\mathrm{BH}$ in the original location with the latest high voltage and vacuum modifications.

The BBQ hardware is in good shape, stable and well documented. Software improvements are being implemented to make operation easier. The goal is to simplify and automate as much as possible but 'experts' will still be needed. 4 more experts are now available and they have been able to support APEX in Run-7.

A Traveling Wave Cavity was tested as an abort gap monitor: the results are promising but some of the data has been confusing. There have been times that the spectra seem to show more beam in the gap(s) than where it is expected. Work is in progress.

After Phase 3B of the cryogenics system upgrade the power consumption stabilized at slightly over $5 \mathrm{MW}$ in physics operation. That is a significant improvement over last year's $\sim 6 \mathrm{MW}$. The 3 phases of the cryo system upgrade over 5 years in fact decreased power consumption by almost $50 \%$. 
This is what was done in phase 3 in more details:

- New gas bearing turbine for energy removal at the cold end of the refrigerator (Run-7).

- New high efficiency vertical heat exchanger system at the cold end of refrigerator (Run-7).

- Re-configured the cold helium supply to the accelerator rings to eliminate the use of the cold circulators (Run-6).

- Modified Cold Box 5 to reduce Helium inventory, improve insulation, and reduce flow restrictions (Run-6).

Aside from the power savings these measures also reduced the liquid inventory in the refrigerator and reduced the number of running compressors by $4 \mathrm{FS}$ and 1 SS.

These improvements did not come entirely or free: at the beginning of the run the oil contamination in the heat exchanger HX20 caused several almost 2 weeks of interruption of operation (not entirely reflected in the operations statistics). A bypass of the affected exchanger efficiently solved the problem. Other than the HX20, Cryogenics down time was about 13 hours, 8 of which were due to failed PLC cards. A major event (6.5 hour down) was a failed PLC Analog output card in the Compressor room. A failed card caused power supply to crowbar. This ultimately affected other PLC analog cards since they did not have power. The ALPHA Computers and New Control Software for Turbine 7 worked very well during the run with the exception of an Alpha that failed at the end of the run without the backup kicking in).

Cryogenics planned improvements include:

- A draft of a Long Term Upgrade Plan for Cryo Instrumentation \& Control has been written, needed also for the planned CCR and MCR integration.

- Replacement of near obsolete equipment.

- Improved brake control for the T5 and T6 turbines will be installed this summer (metering valves). This will allow these turbines to run at higher speed with lower flow. (Phase IIIC - tuned during 2008 run).

- New operating flow path in RHIC for the $80 \mathrm{~K}$ cooler to reduce LN2 consumption.

- He system leaks

Only 4 trouble reports were filed this run on account of the vacuum system, all involving either AtR valve solenoids or leaks of the BtA multiwires bellows. All events were analyzed: the major cause of valve solenoid failures is water in air lines while the multiwires bellows have a very high spring rate and are nearly collapsed when the multiwire is fully inserted.

The plan for shutdown work is the following:

- Install NEG in remaining warm bores and in Brahms

- Install new Compressor and Desiccant dryer in 1000P for ATR line

- Replace all valve solenoids- clean and dry all regulators/lines

- Upgrade all RHIC air regulators with water separator/filters

- Complete AGS ion pump upgrade A-10 house

- Start AGS gauge and controller system upgrade

- Install Digis in place of communication co-processors in RHIC houses

- Install new bellows on BTA multiwire with low spring rate 
- Install cryo-pumps in RF at SWC for faster pump-downs

\section{New developments and future plans}

The search for the critical point in the phase diagram of the quark-gluon plasma has motivated RHIC operation at energies below the nominal injection energy. Operation at half the injection rigidity was tested during Run- 6 with protons and during Run-7 with gold beams. Both tests were successful. RF acceptance, intra-beam scattering, and field quality are acceptable. For gold beams the RF harmonic needed to be changed to 366 due to the lower velocity resulting in unexpected problems with the PHENIX data acquisition system. STAR was able to take data. Preliminary analysis gave an approximate peak luminosity of $0.4 \times 10^{24} \mathrm{~cm}^{-2} \mathrm{~s}^{-1}$, which is larger than expected from $\gamma^{2}$ scaling. Based on the success of these test runs an additional test run at $1 / 4$ of the injection energy could be scheduled for the next run and a low energy data run could be scheduled for run- 9 .

At the lowest energy the RF matching between AGS and RHIC will require a smaller longitudinal emittance than presently achieved. Electron cooling in the AGS was considered to reduce the longitudinal emittance. However, during the injector session of the retreat Leif Ahrens showed that with the new "Sandwich" stripping foil in the BTA it should be possible to achieve the required smaller longitudinal emittance of $0.08 \mathrm{eVs} / \mathrm{n}$ by longitudinally damping the coherent bunch motion using the L10 broadband cavity.

For regular operation of RHIC at $100 \mathrm{GeV} / \mathrm{n}$ Intra-Beam Scattering (IBS) is limiting the achievable luminosity. IBS leads to an increase in the longitudinal emittance and eventually to beam debunching. Through dispersion it also leads to increased horizontal emittance and with coupling to vertical emittance growth. The transverse emittance growth reduces the luminosity directly. During Run-7 a lattice with reduced dispersion was successfully tested. The lower dispersion was achieved by increasing the betatron tunes by three units. With such a lattice the transverse emittance growth should be reduced and $20-30 \%$ more luminosity could be achievable during gold-gold running.

During Run-7 first efforts were made to counteract IBS directly. Longitudinal bunched beam stochastic cooling was demonstrated for the first time with $100 \mathrm{GeV} / \mathrm{n}$ gold beams. The stochastic cooling of the yellow beam increased the luminosity lifetime to give about $15 \%$ more integrated luminosity and also narrowed the vertex distribution. Longitudinal stochastic cooling in the blue beam is now under construction.

With the success of longitudinal stochastic cooling plans are being made to install a single plane of transverse stochastic cooling in the yellow ring by run-9. If this is successful the remaining three planes for both rings will be installed. (One plane per ring could possibly be enough with coupling). With a full complement of stochastic cooling in RHIC the average store luminosity is predicted to increase to $4 \times 10^{27} \mathrm{~cm}^{-2} \mathrm{~s}^{-1}$, about three times over the $1.2 \times 10^{27} \mathrm{~cm}^{-2} \mathrm{~s}^{-1}$ reached during Run-7.

IBS in RHIC can be completely counteracted with electron cooling. The latest simulations show that with the nominal beam intensity of $1 \times 10^{9}$ gold ions per bunch an average luminosity of $8.5 \times 10^{27} \mathrm{~cm}^{-2} \mathrm{~s}^{-1}$ can be reached. Electron cooling remains 
effective even with a beam intensity of $2 \times 10^{9}$ gold ions per bunch giving an average store luminosity of $20 \times 10^{27} \mathrm{~cm}^{-2} \mathrm{~s}^{-1}$. Preparations for the Test ERL are making good progress. The super-conducting Linac cavity has reached the design accelerating gradient of $20 \mathrm{MV} / \mathrm{m}$ and design and construction of the super-conducting RF electron gun has started. The Test ERL is scheduled to be ready for commissioning with beam at the beginning of 2009 .

During rebucketing from the $28 \mathrm{MHz}$ accelerating RF system to the $200 \mathrm{MHz}$ storage RF system part of the gold beam ends up in satellite bunches around the main $5 \mathrm{~ns}$ long bunch. During the store these satellite bunches grow due to IBS. Even with longitudinal stochastic cooling the satellite bunches are not completely eliminated. With a high gradient $56 \mathrm{MHz}$ RF system the rebucketing can be made adiabatic and the much larger bucket area of this RF system will prevent the formation of satellite bunches during the store. A design for a $56 \mathrm{MHz}$ super-conducting cavity was presented that can provide 2 MV based on a reentrant quarter-wave resonator. A single cavity per ring would be sufficient to avoid satellite bunches.

Possible luminosity improvements for proton-proton operation were also discussed. A major limitation for pp luminosity is beam-beam interaction and the tune spread it introduces. Initial simulations show that a low energy electron beam lens can reduce this tune spread to almost the level of no beam-beam interactions. Further simulations are needed to show that the beam lifetime can also be restored. Such an electron lens would look quite similar to the EBIS electron beam.

Finally, since the proton-proton luminosity lifetime is not limited by particle burn-off, as is the case for gold-gold operation, it would be beneficial to reduce beta-star to below the present limit of about $50 \mathrm{~cm}$. This could be achieved with additional quadrupoles close to the interaction point. An initial solution with a quadrupole each after the D0 and DX dipoles yields a beta-star of $34 \mathrm{~cm}$ with a maximum beta function in the triplets of 2200 $\mathrm{m}$.

\section{References}

[1] C.J. Gardner, et al. "Setup and Performance of the RHIC Injector Accelerators for the 2007 Run with Gold Ions", Proceedings of PAC07, Albuquerque, New Mexico, pp.18621864 .

[2.] L.A. Ahrens, et al., "The RHIC Injector Accelerator Configurations, and Performance for the RHIC 2003 Au-d Physics Run", Proceedings of the 2003 Particle Accelerator Conference, pp. 1715-1717.

[3] K.A. Drees, et al., "Summary of the RHIC Performance during the FY07 Heavy Ion Run", Proceedings of PAC07, Albuquerque, New Mexico, pp. 722-724.

[4] T. Satogata, et al., "Commissioning of the RHIC Deuteron-Gold Collisions", Proceedings of the 2003 Particle Accelerator Conference, pp.1706-1708. 